\title{
Conocimiento sobre el Consumo de Sustancias Psicoactivas en Estudiantes de Enfermería
}

\section{Knowledge Based on Psicoactives Substances Consumption in Nursing Students}

\author{
Gloria Lastre Amell ${ }^{1}$ \\ Universidad Simón Bolívar \\ Gladys Gaviria García ${ }^{2}$ \\ Universidad Simón Bolívar \\ Nancy Arrieta Reales ${ }^{3}$ \\ Universidad Simón Bolívar
}

\section{RESUMEN}

Introducción: Las sustancias psicoactivas son capaces de alterar el organismo y ejercen su acción sobre la conducta, percepción y conciencia de los individuos. El consumo excesivo, persistente y periódico de las mismas se define como dependencia. El conocimiento de tales sustancias y de la dependencia resulta fundamental para las personas y, en particular, para los jóvenes. Objetivo: Identificar el conocimiento que poseen los estudiantes de enfermería de una institución privada respecto a las sustancias psicoactivas lícitas e ilícitas (SPA). Materiales y métodos: Se realizó un estudio cuantitativo en 220 estudiantes representativos de todos los grados del programa de enfermería referido. Para ello, se utilizó un muestreo aleatorio simple, y a los seleccionados seles aplicóun instrumento que evaluó edad, sexo, conocimientos, órganos afectados y sustancias perjudiciales. Resultados: El 81\% de los estudiantes era de sexo femenino, y sus edades comprendían entre 17 y 21 años.El 99,6\% consideró que el consumo de sustancias psicoactivasson perjudiciales, en tanto que el $95 \%$ estimó que el cerebro es el órgano más afectado por el consumo. El 9,5\% consideró que el alcohol no es una droga depresora, y el 18\% afirmó que tampoco la heroína lo es. Las benzodiacepinas constituyen la sustancia que en mayor proporción (83\%) fue seleccionada como estimulante. Sólo el $26 \%$ considera que el LCD es una sustancia alucinante. Conclusión: Los estudiantes reconocen las sustancias psicoactivas como perjudiciales para la salud, pero pocos muestran conocimientos sobre su clasificación.

Palabras Clave: conocimiento, consumo, sustancias psicoactivas (Fuente: DeCS).

\begin{abstract}
Introduction: Psychoactive substances have the potential to alter the body and they act on the behavior, perception and consciousness of individuals. Their excessive, persistent and periodical use is defined as dependence. Awareness of these substances and dependence on them is essential for people and, in particular, for the youth. Objective: To identify the level of awareness of legal and illegal psychoactive substances (PASs) among nursing students of a private institution. Materials and Methods: A quantitative study was carried out on 220 students representing each level of the nursing program mentioned. To achieve this, a simple random sample was used, and an instrument was given to the selected students which assessed age, sex, knowledge, affected organs and harmful substances. Results: $81 \%$ of the students were females between the ages of 17 and 21 years. $99.6 \%$ considered that the use of psychoactive substances is harmful, as $95 \%$ estimated that the brain is the most affected organ. 9.5\% thought that alcohol was not a depressant drug, and $18 \%$ stated the same about heroin. Benzodiazepine was the substance which in large part (83\%) was chosen as a stimulant. Only $26 \%$ thought that LCD was a hallucinatory substance. Conclusion: The students recognized psychoactive substances as harmful to health, but few showed knowledge of its classification.
\end{abstract}

Keywords: awareness, use, psychoactive substances (Source: MeSH, NLM).

\footnotetext{
${ }^{1}$ Enfermera. MSc en Salud Pública. Correo electrónico: glastre@unisimonbolivar.edu.co

${ }^{2}$ Enfermera. MSc en Educación. Correo electrónico: ggaviria1@unisimonbolivar.edu.co

${ }^{3}$ Enfermera. MSc en Educación. Correo electrónico: narrieta1@unisimonbolivar.edu.co
} 


\section{Introducción}

Las sustancias psicoactivas (SPA) son aquellas drogas cuyo consumo puede producir dependencia, estimulación o depresión del sistema nervioso central, o que producen un trastorno en la función del juicio, el comportamiento o el ánimo de la persona (1). También merece esta denominación toda sustancia capaz de alterar el organismo y de ejerceracción psíquicasobre la conducta, la percepción y la conciencia. El sometimiento de la droga es el consumo excesivo, continuo y periódico de una sustancia tóxica (2).

La Organización Mundial de la Salud (OMS) considera como SPA a toda sustancia que al ser introducida en el organismo, por cualquier vía de administración, produce una alteración en el natural funcionamiento del Sistema Nervioso Central del individuo y es, además, susceptible de crear dependencia, ya sea psicológica, física o ambas (3). La dependencia de SPA es multifactorial yestá determinada por factores biológicos y genéticos. De modo que los caracteres hereditarios pueden desempeñar un papel importante en el consumo, junto a factores psicosociales, culturales y ambientales (4).

Asimismo, como anota Hall (5), las SPA son sustancias químicas que interfieren en la comunicación del cerebro y afectan la manera en que las células nerviosas envían y reciben los mensajes. En este sentido, se sabe que el cerebro contiene docenas de diferentes tipos de receptores y de mensajeros químicos, y que las áreas de éste que resultan más afectadas por el uso de drogas son: el tallo, que controla funciones básicas; el sistema límbico, que contiene el circuito de gratificación, la capacidad de sentir placer; y, por último, la corteza, que controla funciones como ver, sentir, oír y saborear, además de los pensamientos.

Por otra parte, distintas organizaciones y expertos en SPA, como la Organización Mundial de la Salud (OMS), la Comisión Interamericana para el Control del Abuso de Drogas (CICAD/OEA), la Asociación Americana de Medicina Adictiva (ASAM), las comisiones nacionales de drogas de otras naciones y la propia experiencia desarrollada en Colombia por distintos expertos, así como por organizaciones gubernamentales y no gubernamentales a través de varias décadas, se han ocupado de la problemática del conocimiento de consumo de sustancias psicoactivas (6).
Según el Observatorio de Drogas de Colombia (ODC), el conocimiento del fenómeno de las drogas se presenta de manera muy diferenciada entre los universitarios, lo que indica la necesidad de mantener la enseñanza respecto a la integración, acción y conocimientos referentes a lasdrogas y a las políticas y programas nacionales e internacionales. Así está descrito en las acciones y líneas que enfocan la salud internacional y el fenómeno de las drogas como una fortaleza para mejorar la intervención en el área de salud (7).

Por otro lado, al revisar estudios que evalúan el conocimiento, consumo y la prevalencia del uso de alcohol, tabaco y drogas entre estudiantes, se identificó que el consumo de drogas se ha incrementado a los largo de los años, debido al déficit de conocimiento de los estudiantes acerca de las drogas licitas e ilícitas y los efectos adversos que estas ocasionan a los consumidores (8).

Por tanto, esto crea una problemática de las drogas, que debe ser atendida como un tema de salud pública, a fin de controlarlo periódicamente y contrastarlo con las políticas públicas para medir su eficacia y eficiencia. Ello exige una base sólida de conocimiento científico y terapéutico para que se logre el éxito. En esta orientación, los profesionales de la salud deben tener en cuenta esta demanda social y aportar al conocimiento científico para enfrentar los riesgos de este mal, a través de una adecuada calidad de atención primaria en salud $(9,10)$.

En un estudio sobre el conocimiento de las drogas en estudiantes y docentes del área de medicina, realizado en La Paz, Bolivia, se demostró que el patrón de consumo de alcohol en la población de universitarios es excesivo, y,del mismo modo, entre estos se presenta el uso experimental de drogas ilícitas. Debe tenerse claro que la principal razón por la que estas sustancias se constituyen en un problema sanitario no es el riesgo de muerte, sino su capacidad para generar altos niveles de dependencia que alteran el desarrollo vital de la persona, ocasionando no solo degeneraciones en su estado de salud y a nivel afectivo, económico y social $(9,11)$.

De acuerdo con la Comisión Interamericana para el Control del Abuso de Drogas (CICAD), las sustancias psicoactivas se dividen en cuatro grupos: depresores, estimulantes, alucinógenos y sustancias mixtas. Las depresores disminuyen el nivel de funcionamiento del 
sistema nervioso central. En este grupo se encuentran: elalcohol, narcóticos como el opio, la morfina y la heroína, e inhalables como la gasolina, acetona, pegantes. Los estimulantes se definen comosustancias que aumentan la agudeza psicológica y la capacidad física. Entre estos tenemos: la cocaína, el bazuco, las anfetaminas y la cafeína. Los alucinógenos, por su parte, tienen la capacidad de producir alteraciones en la percepción. Por último, están las sustancias mixtas, producen efectos combinados, es decir, pueden disminuir o acelerar el nivel de funcionamiento del sistema nervioso central. Dentro de este grupo se encuentran la marihuana y el éxtasis (12).

Existen síntomas del uso y abuso de SPA que pueden ser reconocidos como señales de peligro. Los síntomas más persistentes en el consumo son: ojos enrojecidos, tos persistente, pupilas dilatadas, puntiformes; nistagmos, signo de consumo de sustancias psicoactivas; inapetencia relacionada con anfetaminas, metanfetaminas y cocaína; aumento del apetito asociada con el consumo de marihuana; apatía o somnolencia constante relacionada con drogas opiáceas como la heroína, codeína o con el efecto de atenuación del consumo de drogas estimulantes; hiperactividad asociada con la cocaína y las metanfetaminas (13).

En este orden de ideas, el conocimiento definido como el conjunto de información almacenada mediante la experiencia o el aprendizaje, o a través de la introspección, favorece conductas adecuadas de promoción de salud y es un factor determinante para adoptar hábitos saludables.

Por todo lo planteado hasta aquí, el objetivo del trabajo del que resultó el presente artículo consistió en identificar el conocimiento de los estudiantes de enfermería de una universidad privada de la ciudad de Barranquilla sobre sustancias psicoactivas lícitas e ilícitas.

\section{MATERIALES Y MÉTODOS}

Se realizó un estudio descriptivo transversal en 220 estudiantes de primer semestre y estudiantes de énfasis de un programa de Enfermería. Este programa contaba con una población total de 800 estudiantes, y los participantes fueron seleccionados por muestreo aleatorio simple y de acuerdo con el curso académico.
La recolección de datos fue realizada en el periodo comprendido entre marzo y abril de 2010.

Los criterios de inclusión fueron dos: ser estudiantes de enfermería de la universidad privada y firmar en forma voluntaria el consentimiento informado. Se aplicó una encuesta diseñada y validada por el Dr. Jairo Cepeda, toxicólogo de la Universidad de Norte, quien autorizó su uso en la investigación. La encuesta constó de 37 preguntas de selección múltiple, distribuidas en cuatro partes. La primera parte indaga sobre la características sociodemográficas. Un segundo aspecto lo constituyó el conocimiento, en el cual se ubicó la clasificación de sustancias psicoactivas, en consideración asi eran estimulantes, depresivas, o alucinógenas. Otro elemento indagó si las sustancias producen efectos perjudiciales para la salud, esta se respondieron en forma dicotómica. Por último, había un grupo de preguntas para identificar los órganos del cuerpo que se afectan por el consumo y los efectos secundarios que presentan los individuos bajo el efecto de sustancias psicoactivas.

Antes de la recolección de la información, se explicó a los estudiantes el objetivo del estudio, así como principios éticos, confidencialidad, veracidad de la información y derecho a retirarse cuando la persona determinara. Se obtuvo el consentimiento informado escrito para participar. La información fue analizada en el programa SPSS V.18, permitiendo establecer la frecuencia y los porcentajes correspondientes a cada variable de estudio.

\section{RESULTADOS}

El $81 \%$ de los estudiantes era de género femenino y el 19\%, masculino. La mayor población se encontraba en la etapa adolescente, entre 18 a 21 años, pero se aclara que en la población femenina, se evidencia mayor número de adolescentes, mientras que en el grupo masculino predominan edades de adultos jóvenes. 


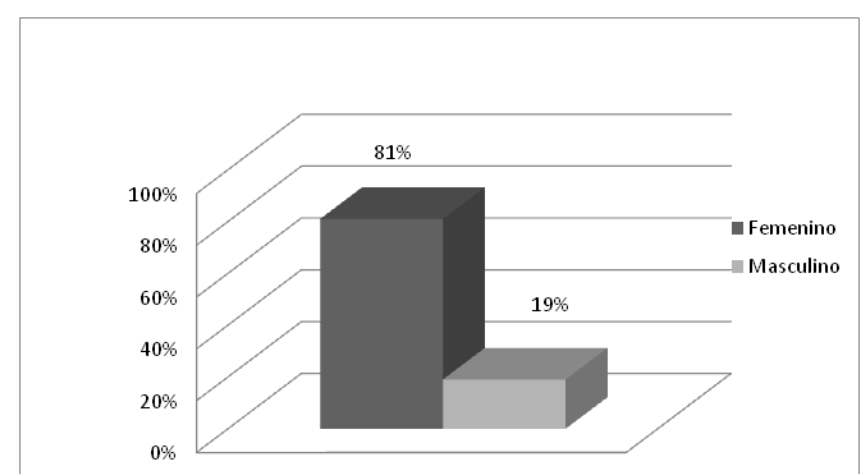

Figura 1. Sexo de los sujetos estudiados

En atención a la clasificación de las sustancias psicoactivas, llama la atención que un porcentaje mínimo de estudiantes, 9,5\% y18\%, considera que el alcohol y la heroína, respectivamente, no son drogas depresoras. Igualmente, se evidencióque las benzodiacepinas constituyen la sustancia que fue seleccionada como estimulanteen mayor proporción (83\%). Sólo el $26 \%$ de los sujetos participantes considera que el LCD es una sustancia alucinante.

En relación con las sustancias mixtas, un porcentaje alto de estudiantes $(60 \%)$ escogióa la marihuana y al éxtasis como drogas que se combinan con otras sustancias para lograr un mayor efecto (Tabla 1).

TABLA 1. Conocimiento de las clasificación de las sustancias psicoactivas

\begin{tabular}{lcc}
\hline \multicolumn{1}{c}{ SUSTANCIAS } & Frecuencia & Porcentaje \\
\hline ALCOHOL & & \\
\hline Depresor & 21 & 9,5 \\
No depresor & 199 & 90,5 \\
\hline HEROÍNA & 39 & 18 \\
\hline Depresor & 181 & 82 \\
No depresor & & \\
\hline INHALABLES & 67 & 30,4 \\
\hline Depresor & 153 & 69,6 \\
No depresor & & \\
\hline COCAÍNA & 69 & 31,3 \\
\hline Estimulante & 151 & 68,7 \\
No estimulante & & \\
\hline BAZUCO & 51 & 23,1 \\
\hline Estimulante & 169 & 76,9 \\
No estimulante & & \\
\hline BENZODIACEPINAS & 37 & 17 \\
\hline Estimulante & 183 & 83 \\
No estimulante & & \\
\hline ANFETAMINAS & 53 & 24 \\
\hline Estimulante &
\end{tabular}

\begin{tabular}{lcc}
\hline \multicolumn{1}{c}{ SUSTANCIAS } & Frecuencia & Porcentaje \\
\hline No estimulante & 167 & 76 \\
\hline CIGARRILLO & & \\
\hline Estimulante & 95 & 43 \\
No estimulante & 125 & 57 \\
\hline LSD & & \\
\hline Alucinógena & 57 & 26 \\
No Alucinógena & 163 & 74 \\
\hline MARIHUANA & & \\
\hline Sustancia mixta & 139 & 63 \\
No Sustancia mixta & 81 & 37 \\
\hline EXTASIS & & \\
\hline Sustancia mixta & 112 & 60 \\
No Sustancia mixta & 108 & 40 \\
\hline
\end{tabular}

Con relación a la variable que indagaba si las sustancias psicoactivas son perjudiciales, el 99,6\% considera que sí lo son. Y en referencia a los órganos más afectados por los consumidores de SPA, el 95\% respondió que el cerebro es el órgano más afectado. Por otro lado, el síntoma más relevantes según los encuestados es la hiperactividad, con un 90\% (Tabla 2).

Tabla 3. Conocimiento sobre las sustancias psicoactivas: perjudiciales, órganos afectados y síntomas CONOCIMIENTO

\begin{tabular}{lcc}
\hline $\begin{array}{l}\text { Perjudiciales } \\
\text { para la salud }\end{array}$ & FRECUENCIA & PORCENTAJES \\
\hline Si & 219 & $99.6 \%$ \\
No & 1 & 0.45 \\
& \\
\hline $\begin{array}{l}\text { Organos del cuerpo que afecta a la salud las sustancias } \\
\text { psicoactivas }\end{array}$ & \\
\hline Cerebro & 209 & $95 \%$ \\
Pulmones & 203 & $92 \%$ \\
Corazón & 197 & $89.5 \%$ \\
& & \\
\hline Síntomas presentes & & $90 \%$ \\
\hline Hiperactividad & 198 & $72.7 \%$ \\
Fuma & 160 & $80 \%$ \\
Ojos rojos & 156 & \\
\hline
\end{tabular}

\section{Discusión}

En principio, se pudo observar que el promedio de edades estuvo entre los 17 a 21 años, con un $86 \%$. En cuanto a la variable de conocimiento sobre la clasificación de las sustancias psicoactivas, el estudio expresó que los estudiantes sí conocen la clasificación, 
lo cual coincide con el estudio de Navia (9), donde un $82 \%$ de los estudiantes universitarios del estudio reconocen qué son drogas psicotrópicas.

De igual manera, se evidenció que para un alto porcentaje de estudiantes las SPA son perjudiciales para la salud, contrastando estos resultados con el estudio del consenso científico sobre sustancias psicoactivas tabaco, alcohol y sustancias ilegales de la Organización Mundial de la Salud, en el que se refiere que las sustancias psicoactivas constituyen un lastre notable para la sociedad en términos de salud. En particular, el tabaco y el alcohol se encuentran entre las principales causas de muerte y discapacidad en los países desarrollados. Además, el consumo mundial de SPA y de otras sustancias controladas está aumentando rápidamente, lo que contribuye de forma significativa a la carga mundial de morbilidad (14).

Un estudio de conocimiento, actitudes y prácticas con el consumo de sustancias psicoactivas en estudiantes de una universidad del departamento del Atlántico, en el cual Melina describe que las sustancias psicoactivas constituyen un problema de salud pública muy importante, dado que, por ejemplo, la cirrosis es un típico ejemplo del abuso del alcohol (15), coincide con lo que los estudiantes reconocieron al respecto en nuestra investigación, pues ellos reconocieron que estas sustancias psicoactivas afectan los órganos del cuerpo, siendo el cerebro el más perjudicado.

En cuanto a los síntomas que más identificaron los estudiantes como presentes en personas que se encuentra bajo el efecto de consumo de drogas, se destacaron la hiperactividad, el fumar y los ojos enrojecidos. Estos resultados corroboran el artículo de Gavin (16), quien afirma que cuando una persona consume drogas, se pueden percibir algunos cambios en su aspecto y en su forma de actuar. También se puede presentar algunos signos como perder interés por los estudios, cambiar de amistades (empezar a salir con personas que consumen drogas), volverse variable, negativa $o$ inquieta, o parecer preocupada constantemente y tener los ojos rojos (17).

La población en estudio tiene conocimientos acerca de las sustancias psicoactivas, pero es de resaltar las deficiencias que tienen en la clasificación de las mismas, debido a que un alto porcentaje de estudiantes consideró que el alcohol y el cigarrillo, entre otros, son sustancias no depresoras, estimulantes y alucinógenas.
Por último, en lo que concierne a los órganos más afectados y los síntomas presentes en los individuos que consumen sustancias psicoactivas, los estudiantes mostraron conocimiento e identificaron que el órgano más afectado en los consumidores de sustancias psicoactivas es el sistema nervioso central.

\section{Referencias}

1. Centro de Tirrenia de psicoterapia - Fono 2245502 Clasificación y descripción de las drogas más utilizadas. Disponible en: http:// Www.drogas.cl/drogas.htm

2. Musacchio DA, Ortiz FA. Drogadicción. Buenos Aires: Paidos; 2000.

3. Madrigal R. Habilidades para la Vida: Manual de Capacitación para docentes: Habilidades para la vida: Prevención del Consumo de Tabaco, Alcohol y otras Drogas. Washington: Organización Panamericana de la Salud; 1999.

4. Organización Mundial de la Salud. Neurociencia del consumo y dependencia de sustancias psicoactivas. Washington, D.C: OP; 2005.

5. Hall W, Degenhardt L. Adverse health effects of non-medical cannabis use. Lancet. 2009; 374: 1383-1391.

6. Londoño C, García W, Valencia SC, Vinaccia S. Expectativas frente al consumo de alcohol en jóvenes universitarios colombianos. Anales de Psicología. 2005; 21(2):259-267.

7. Enríquez BJ, Moreno ML, Barrio LIR, Contreras GM, Orozco MR, Mendoza TM. Conocimiento de los estudiantes de secundaria sobre los tipos de drogas que se consumen en su medio. Rev Col Psicol. 2006; 15: 51-55.

8. Presidencia de la Republica de Colombia. Programa Presidencial para Afrontar el Consumo de Drogas. RUMBOS. Juventud y Consumo de Sustancias Psicoactivas: Resultados de la encuesta nacional de 2001 en jóvenes escolarizados de 10 a 24 años. Bogotá. 2002.

9. Navia BP, Farah BJ, Yaksic FN, Philco LP, Takayanagui AM. Conocimiento sobre el fenómeno de las drogas en entre estudiantes y docentes de la Facultad de Medicina Universidad Mayor de San Andrés, La Paz, Bolivia. Rev. 
Latino-Am. Enfermagem. 2011; 19 (Sup): 722729.

10. Wright MGM, Takayanagui AMM, Ventura CAA. Salud Internacional y el fenómeno de las drogas en las Américas. Dimensiones de las relaciones internacionales en el contexto de la globalización, de la salud internacional y de la internacionalización de riesgos. Ribeirão Preto (SP): Escuela de Enfermería de Ribeirão Preto, SENAD; 2007. Modulo I Parte I, p. 1-39.

11. National Institute on Drug Abuse: Preventing drug use among children and adolescents. 1997.

12. Comisión Interamericana para el Control del Abuso de Drogas (CICAD). Organización de los Estados Americanos. Evaluación del progreso del control de drogas. Washington, D.C; 2003. Informe 1-11 Informe Hemisférico.

13. Stager MM. Substance abuse. En: Kliegman RM, Behrman RE, Jenson HB, Stanton BF. Textbook of Pediatrics. 19th ed. Philadelphia: Saunders Elsevier; 2011.

14. Organización Mundial de la Salud OMS. Consenso Científico sobre Sustancias psicoactivas tabaco, alcohol y sustancias ilegales. Ginebra. 2004.

15. Melina CJ, De la Cruz OA, De la Salas RK, Eguis VR, Galves RD, Caro PS, et al. Conocimientos, actitudes y prácticas relacionados con el consumo de sustancias psicoactivas en estudiantes de enfermería de una universidad del departamento del Atlántico (Colombia). Salud UniNorte. 2012; 28(2): 322-334.

16. Gavin LM. Lo que necesitas saber de las drogas y medicamentos. 2010 [Disponible en internet]http://kidshealth.org/kid/en_espanol/sano /know_drugs_esp.html

17. Castro N, Cortés P, Vasters GP, Costa JM. Uso de drogas entre estudiantes de la Facultad de Ciencias Médicas de la Universidad Nacional Autónoma de Nicaragua, León, Nicaragua. Rev. Latino-Am. Enfermagem. 2010; 18 (Spe): 606612. 